\title{
NOTE ON SOME INFINITE PRODUCTS FOR $\pi$
}

\author{
Peter Kahlig ${ }^{1}$, Janusz Matkowski ${ }^{2}$

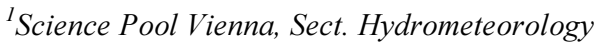 \\ Vienna, Austria \\ ${ }^{2}$ Faculty of Mathematics, Computer Science and Econometrics \\ University of Zielona Góra, Zielona Góra, Poland \\ IPeter.Kahlig@gmx.at, ${ }^{2}$ J.Matkowski@wmie.uz.zgora.pl
}

\begin{abstract}
After a brief review of (slowly converging) Wallis-type infinite products for $\pi$, (faster converging), Dido-type infinite products for $\pi$ are treated. The notion of "alternating products" facilitates error checking.
\end{abstract}

Keywords: infinite product, Wallis-type product, Dido functional equation, Dido sequence, algebraic number, transcendental number, constructible number

Mathematics Subject Classification: Primary 40A20; Secondary 39B12

Dedicated to Ludwig Reich on the occasion of his $75^{\text {th }}$ birthday

\section{Introduction}

In Section 1 we review Wallis-type infinite product representations of $\pi$. In Section 2 we touch on the Dido functional equation, which is used in Section 3 to construct convenient Dido-type infinite product representations of $\pi$. Computational aspects are treated in Section 4 to certify that (contrary to some opinions among physicists) infinite products may be useful even in numerical work. The notion of "alternating products" (Section 3) facilitates error checking in Section 4.

\section{Wallis-type infinite product representations of $\pi$}

Wallis' famous infinite product (originally obtained by an interpolation process, cf. $[1,2])$ reads

$$
\mathrm{W}=\frac{3 \cdot 3 \cdot 5 \cdot 5 \cdot 7 \cdot 7 \cdots}{2 \cdot 4 \cdot 4 \cdot 6 \cdot 6 \cdot 8 \cdots}, \quad \text { where } \quad W=\frac{4}{\pi}
$$


Taking reciprocals, this is equivalent to

$$
\begin{gathered}
\frac{\pi}{4}=\frac{2 \cdot 4}{3 \cdot 3} \cdot \frac{4 \cdot 6}{5 \cdot 5} \cdot \frac{6 \cdot 8}{7 \cdot 7} \Lambda=\prod_{k=1}^{\infty} \frac{2 k \cdot(2 k+2)}{(2 k+1)^{2}}=\prod_{k=1}^{\infty} \frac{4 k \cdot(k+1)}{(2 k+1)^{2}} \\
=\prod_{k=1}^{\infty}\left(1-(2 k+1)^{-2}\right)=\left(1-3^{-2}\right) \cdot\left(1-5^{-2}\right) \cdot\left(1-7^{-2}\right) \Lambda=\frac{8}{9} \cdot \frac{24}{25} \cdot \frac{48}{49} \Lambda .
\end{gathered}
$$

Let us call (1) "Wallis' first product". Multiplying by 2 (and grouping carefully to maintain a distinct formation law) leads to another version, now generally called "Wallis' product" (cf. [3, 4]) or, more properly, "Wallis' second product",

$$
\begin{gathered}
\frac{\pi}{2}=\frac{2 \cdot 2}{1 \cdot 3} \cdot \frac{4 \cdot 4}{3 \cdot 5} \cdot \frac{6 \cdot 6}{5 \cdot 7} \Lambda=\prod_{k=1}^{\infty} \frac{(2 k)^{2}}{(2 k-1)(2 k+1)}=\prod_{k=1}^{\infty} \frac{(2 k)^{2}}{(2 k)^{2}-1} \\
=\prod_{k=1}^{\infty}\left(1-(2 k)^{-1}\right)^{-1}=\left(1-2^{-2}\right)^{-1} \cdot\left(1-4^{-2}\right)^{-1} \cdot\left(1-6^{-2}\right)^{-1} \Lambda=\frac{4}{3} \cdot \frac{16}{15} \cdot \frac{36}{35} \Lambda
\end{gathered}
$$

Remark 1. Of course, instead of (2), $\pi / 2$ could be expressed by doubling (1),

$$
\begin{aligned}
\frac{\pi}{2} & =2 \frac{\pi}{4}=2 \frac{2 \cdot 4}{3 \cdot 3} \cdot \frac{4 \cdot 6}{5 \cdot 5} \cdot \frac{6 \cdot 8}{7 \cdot 7} \Lambda=2 \prod_{k=1}^{\infty}\left(1-(2 k+1)^{-2}\right) \\
& =2\left(1-3^{-2}\right) \cdot\left(1-5^{-2}\right) \cdot\left(1-7^{-2}\right) \Lambda=2 \frac{8}{9} \cdot \frac{24}{25} \cdot \frac{48}{49} \Lambda
\end{aligned}
$$

(with a leading scale factor of 2 before the main product with formation law). This is different from the (vanishing) divergent product

$$
\begin{aligned}
& \frac{2 \cdot 2}{3 \cdot 3} \cdot \frac{4 \cdot 4}{5 \cdot 5} \cdot \frac{6 \cdot 6}{7 \cdot 7} \Lambda=\prod_{k=1}^{\infty} \frac{(2 k)^{2}}{(2 k+1)^{2}}=\prod_{k=1}^{\infty}\left(1+(2 k)^{-1}\right)^{-2} \\
= & \left(1+2^{-1}\right)^{-2} \cdot\left(1+4^{-1}\right)^{-2} \cdot\left(1+6^{-1}\right)^{-2} \Lambda=\frac{4}{9} \cdot \frac{16}{25} \cdot \frac{36}{49} \Lambda=0,
\end{aligned}
$$

and different from the (indefinitely growing) divergent product

$$
\begin{aligned}
& \frac{2 \cdot 2}{1 \cdot 1} \cdot \frac{4 \cdot 4}{3 \cdot 3} \cdot \frac{6 \cdot 6}{5 \cdot 5} \Lambda=\prod_{k=1}^{\infty} \frac{(2 k)^{2}}{(2 k-1)^{2}}=\prod_{k=1}^{\infty}\left(1-(2 k)^{-1}\right)^{-2} \\
= & \left(1-2^{-1}\right)^{-2} \cdot\left(1-4^{-1}\right)^{-2} \cdot\left(1-6^{-1}\right)^{-2} \Lambda=\frac{4}{1} \cdot \frac{16}{9} \cdot \frac{36}{25} \Lambda=\infty .
\end{aligned}
$$




\section{The Dido functional equation}

Suppose that a continuous function $f:[2, \infty) \rightarrow[0, \infty)$ satisfies the Dido functional equation

$$
2 f(2 x)=f(x)+\sqrt{f(x)^{2}+\frac{1}{x^{2}}}, \quad x \geq 2,
$$

related to the ancient isoperimetric problem of Dido (cf. [5]). In [6] it is shown that if the constant $\frac{1}{\pi}$ is the asymptote of $f$ at infinity, that is if $\lim _{x \rightarrow \infty} f(x)=\frac{1}{\pi}$, then

$$
f(x)=\frac{1}{x} \cot \left(\frac{\pi}{x}\right), \quad x \in[2, \infty)
$$

Restricting $x$ to integer values, we have the Dido sequence $f(n)=\frac{1}{n} \cot \left(\frac{\pi}{n}\right)$ for $n=2,3,4, \ldots$, and we may interpret $f(n)$ as the inner radius $r_{n}$ (or area $A_{n}$ ) of a regular polygon of order $n$ with fixed perimeter $P$, scaled by half-perimeter $P / 2$

$$
f(n)=r_{n} /(P / 2)=A_{n} /(P / 2)^{2} .
$$

This yields explicitly

$$
\begin{aligned}
& r_{n}=\frac{P}{2} f(n)=\frac{P}{2 n} \cot \left(\frac{\pi}{n}\right), n=2,3,4, \ldots \text { (polygons), with } r_{\infty}=\frac{P}{2 \pi}(\text { circle }) ; \\
& A_{n}=\frac{P^{2}}{4} f(n)=\frac{P^{2}}{4 n} \cot \left(\frac{\pi}{n}\right), n=2,3,4, \ldots \text { (polygons), with } A_{\infty}=\frac{P^{2}}{4 \pi}(\text { circle). }
\end{aligned}
$$

Alternatively, if the outer radius $R_{n}$ of a regular polygon of order $n$ is to be used, the Dido sequence becomes $f(n)=\left(R_{n} \cos \left(\frac{\pi}{n}\right)\right) /(P / 2)$, leading to the expression

$$
R_{n} \cos \left(\frac{\pi}{n}\right)=\frac{P}{2} f(n)=\frac{P}{2 n} \cot \left(\frac{\pi}{n}\right)
$$

implying 


$$
R_{n}=\frac{P}{2 n \sin \left(\frac{\pi}{n}\right)}, n=2,3,4, \ldots \text { (polygons), with } R_{\infty}=\frac{P}{2 \pi}(\text { circle })
$$

The inequality $f(n) \leq \frac{1}{\pi}$ for $n=2,3,4, \ldots$ resembles the well-known isoperimetric inequality (cf. [7]),

$$
r_{n} \leq \frac{P}{2 \pi} \text { or } A_{n} \leq \frac{P^{2}}{4 \pi}, n=2,3,4, \ldots,
$$

where equality holds for $n \rightarrow \infty$ (circle).

\section{Dido-type infinite product representations of $\pi$}

It is convenient (cf. [7]) to use the following:

Definition 1. An algebraic number is called constructible if it is an aggregate of finitely many rationals and/or square roots.

Remark 2. It is well known (cf. [7, 8]) that a regular n-gon is constructible by ruler and compass if and only if its Dido value $f(n)$ ( $n$ fixed) is a constructible algebraic number. (Otherwise the n-gon is not constructible; its Dido value might contain, for instance, a cube root.)

Remark 3. Utilizing well-known product representations of $\cos$ and $\sin$ to form $\cot =\cos / \sin$, we obtain

$$
\pi f(n)=\frac{\pi}{n} \cot \left(\frac{\pi}{n}\right)=\prod_{k=1}^{\infty} \frac{1-((2 k-1) n / 2)^{-2}}{1-(k n)^{-2}}, \quad n=2,3,4, \ldots
$$

Obviously, this expression is an "alternating product"; explicitly, it may be written

$$
\pi f(n)=\prod_{j=1}^{\infty}\left(1-(j n / 2)^{-2}\right)^{(-1)^{j+1}}, \quad n=2,3,4, \ldots
$$

Remark 4. In analogy to Leibniz's well-known criterion for (conditionally convergent) alternating series [namely, the remainder of an alternating series has the sign of the first neglected term, and is closer to 0 than the first neglected term], we may formulate a criterion for alternating products: the remainder of an 
alternating product is $>1$ or $<1$ just like the first neglected factor, and is closer to 1 than the first neglected factor.

Remark 5. Using (10), we can compile values of the Dido sequence $f(n)$ for regular polygons of order $n=2,3, \ldots, 20$. Constructible $n$-gons are marked by *

Order Dido value num.

$$
n \quad f(n)=\frac{1}{n} \cot \left(\frac{\pi}{n}\right) \quad \text { of } f(n)
$$

$\begin{array}{ccc}2^{*} & 0 & 0 \\ 3^{*} & \frac{1}{3 \sqrt{3}} & 0.1925 \\ 4^{*} & \frac{1}{4} & 0.2500 \\ & \frac{\sqrt{5+2 \sqrt{5}}}{5 *} & 0.2753 \\ & \frac{1}{5 *} & \\ 6^{*} & \frac{1}{2 \sqrt{3}} & \\ 7 & f(7) & 0.29687 \\ 8^{*} & \frac{1+\sqrt{2}}{8} & 0.3018 \\ & f(9) & 0.3053 \\ 9 & \end{array}$

9

$10 *$

11

$12 * \quad \frac{2+\sqrt{3}}{12}$

13

14

0

0.3078

0.3096

0.3110

0.3121 related infinite product for $\pi$

$$
\pi f(n)
$$

$$
\frac{\pi}{3 \sqrt{3}}=\frac{1-(3 / 2)^{-2}}{1-3^{-2}} \cdot \frac{1-(9 / 2)^{-2}}{1-6^{-2}} \cdot \frac{1-(15 / 2)^{-2}}{1-9^{-2}} \Lambda
$$$$
\frac{\pi}{4}=\frac{1-2^{-2}}{1-4^{-2}} \cdot \frac{1-6^{-2}}{1-8^{-2}} \cdot \frac{1-10^{-2}}{1-12^{-2}} \Lambda
$$

$\frac{\pi \sqrt{5+2 \sqrt{5}}}{5 \sqrt{5}}=\frac{1-(5 / 2)^{-2}}{1-5^{-2}} \cdot \frac{1-(15 / 2)^{-2}}{1-10^{-2}} \cdot \frac{1-(25 / 2)^{-2}}{1-15^{-2}} \Lambda$

$\frac{\pi}{2 \sqrt{3}}=\frac{1-3^{-2}}{1-6^{-2}} \cdot \frac{1-9^{-2}}{1-12^{-2}} \cdot \frac{1-15^{-2}}{1-18^{-2}} \Lambda$

$\pi f(7)=\frac{1-(7 / 2)^{-2}}{1-7^{-2}} \cdot \frac{1-(21 / 2)^{-2}}{1-14^{-2}} \cdot \frac{1-(35 / 2)^{-2}}{1-21^{-2}} \Lambda$

$$
\frac{\pi(1+\sqrt{2})}{8}=\frac{1-4^{-2}}{1-8^{-2}} \cdot \frac{1-12^{-2}}{1-16^{-2}} \cdot \frac{1-20^{-2}}{1-24^{-2}} \Lambda
$$

$\pi f(9)=\frac{1-(9 / 2)^{-2}}{1-9^{-2}} \cdot \frac{1-(27 / 2)^{-2}}{1-18^{-2}} \cdot \frac{1-(45 / 2)^{-2}}{1-27^{-2}} \Lambda$

$$
\frac{\pi \sqrt{5+2 \sqrt{5}}}{10}=\frac{1-5^{-2}}{1-10^{-2}} \cdot \frac{1-15^{-2}}{1-20^{-2}} \cdot \frac{1-25^{-2}}{1-30^{-2}} \Lambda
$$

$$
\pi f(11)=\frac{1-(11 / 2)^{-2}}{1-11^{-2}} \cdot \frac{1-(33 / 2)^{-2}}{1-22^{-2}} \cdot \frac{1-(55 / 2)^{-2}}{1-33^{-2}} \Lambda
$$

$$
\frac{\pi(2+\sqrt{3})}{12}=\frac{1-6^{-2}}{1-12^{-2}} \cdot \frac{1-18^{-2}}{1-24^{-2}} \cdot \frac{1-30^{-2}}{1-36^{-2}} \Lambda
$$

$$
\pi f(13)=\frac{1-(13 / 2)^{-2}}{1-13^{-2}} \cdot \frac{1-(39 / 2)^{-2}}{1-26^{-2}} \cdot \frac{1-(65 / 2)^{-2}}{1-39^{-2}} \Lambda
$$$$
\pi f(14)=\frac{1-7^{-2}}{1-14^{-2}} \cdot \frac{1-21^{-2}}{1-28^{-2}} \cdot \frac{1-35^{-2}}{1-42^{-2}} \Lambda
$$ 


$$
\begin{aligned}
& \text { 15* } \quad \frac{A}{60} \quad 0.3136 \quad \frac{\pi A}{60}=\frac{1-(15 / 2)^{-2}}{1-15^{-2}} \cdot \frac{1-(45 / 2)^{-2}}{1-30^{-2}} \cdot \frac{1-(75 / 2)^{-2}}{1-45^{-2}} \Lambda, \\
& \text { 16* } \quad \frac{B}{16} \quad 0.3142 \quad \frac{\pi B}{16}=\frac{1-8^{-2}}{1-16^{-2}} \cdot \frac{1-24^{-2}}{1-32^{-2}} \cdot \frac{1-40^{-2}}{1-48^{-2}} \Lambda, \\
& \text { where } A=(1+\sqrt{5})(2 \sqrt{3}+\sqrt{10-2 \sqrt{5}}) \text { and } B=1+\sqrt{2}(1+\sqrt{2+\sqrt{2}}) \\
& \text { 17* } \quad \frac{1}{17} \sqrt{\frac{15+D}{17-D}} \quad 0.3147 \quad \frac{\pi}{17} \sqrt{\frac{15+D}{17-D}}=\frac{1-(17 / 2)^{-2}}{1-17^{2}} \cdot \frac{1-(51 / 2)^{-2}}{1-34^{-2}} \cdot \frac{1-(85 / 2)^{-2}}{1-51^{-2}} \Lambda \text {, } \\
& \text { where } D=\sqrt{17}+\sqrt{34-2 \sqrt{17}}+2 \sqrt{17+3 \sqrt{17}-\sqrt{34-2 \sqrt{17}}-2 \sqrt{34+2 \sqrt{17}}}
\end{aligned}
$$

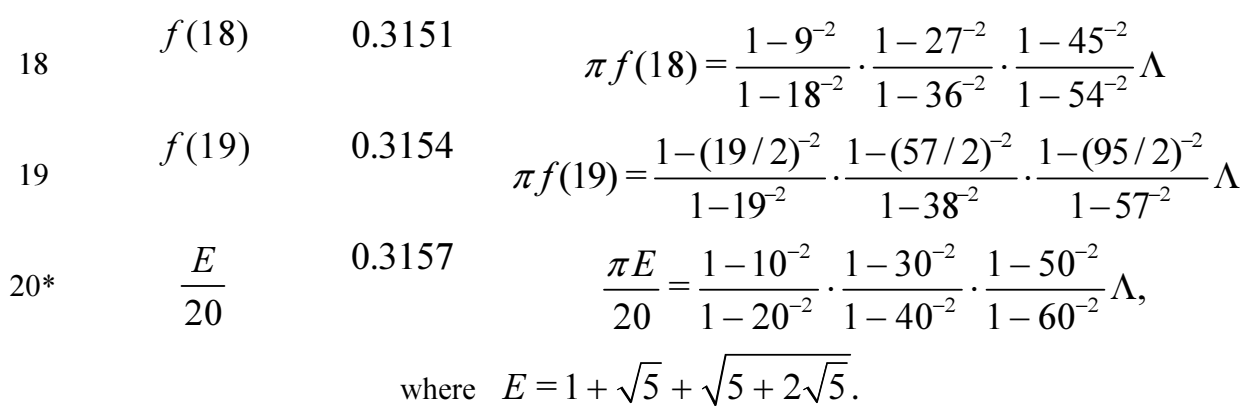

For $n \rightarrow \infty$ we obtain the transcendental number (Dido value for circle)

$$
f(\infty)=\lim _{n \rightarrow \infty} \frac{1}{n} \cot \left(\frac{\pi}{n}\right)=\frac{1}{\pi} \approx 0.3183 .
$$

Remark 6. The items of the list in Remark 5 admit several interpretations. For instance, by (10) we obtain a multitude of infinite products for $\pi$,

$$
\pi=\frac{1}{f(n)} \prod_{k=1}^{\infty} \frac{1-((2 k-1) n / 2)^{-2}}{1-(k n)^{-2}}, \quad n=3,4,5, \ldots,
$$

where $1 / f(n)$ is a scale factor, it is for general $2<n<\infty$ a general algebraic number $>\pi$ [but reasonably simple for $n *$, i.e. for $f(n)$ a constructible algebraic number (cf. Remark 2), which implies in this case that also $1 / f(n)$ (the scale factor) is a constructible algebraic number]. For $2<n<\infty$, the product represents a positive transcendental number $<1$. For $n \rightarrow \infty$, the scale factor approaches $\pi$ while the product approaches 1 . In the extreme case $n=2$, the scale factor grows indefinitely, $1 / f(2)=\infty$, while the product degenerates to 0 . Thus, Dido-type representations of the transcendental number $\pi$ consist in general (namely for $2<n<\infty$ ) of two factors: an algebraic scale factor (of the same magnitude as $\pi$ ) 
and a transcendental infinite product (of magnitude 1). In principle, also Wallistype products like (1) respectively (2) may be interpreted as such a decomposition: $\pi=4 \Pi \ldots$ [used e.g. in (13) below] respectively $\pi=2 \Pi$...; Wallis-type products lack a convenient error estimation (in contrast to Dido-type products, see next section).

\section{Computational aspects}

Approximations (of order $N \in \mathrm{N}$ ) to Wallis's first product (1) may be written

$$
p i(N)=4 \prod_{k=1}^{N}\left(1-(2 k+1)^{-2}\right),
$$

with $p i(\infty)=\pi$. To 3 and 6 significant digits we get

$$
p i(300)=3.14 \ldots \text { and } p i(400000)=3.14159 \ldots,
$$

exhibiting rather slow convergence, and prompting statements like the following [3]: "These infinite products have a variety of uses in analytical mathematics. However, because of rather slow convergence, they are not suitable for precise numerical work". Yet we will show presently that Dido-like infinite products may be numerically useful.

For entry 4 of the list in Remark 5, i.e. taking $n=4$ in (12), we have approximations (of order $N \in \mathrm{N}$ )

$$
\operatorname{Pi}(N)=4 \prod_{k=1}^{N} \frac{1-(4 k-2)^{-2}}{1-(4 k)^{-2}},
$$

with $\operatorname{Pi}(\infty)=\pi$. To 3, 6 and 9 significant digits we obtain here

$$
P i(10)=3.14 \ldots, P i(300)=3.14159 \ldots=\ldots \text { and } P i(10000)=3.14159265 \ldots,
$$

showing an acceptable rate of convergence. Moreover, we can calculate the expected error: according to Remark 4, we just have to look at the first neglected factor $v_{1}$ in (14) [in comparison to (12)], namely $v_{1}(N)=1-(4(N+1)-2)^{-2}$; the remainder $R$ is closer to 1 than $v_{1}$, i.e. $|1-R(N)|<\left|1-v_{1}(N)\right|$, and we obtain for the absolute error $E$ (when retaining $N$ factors only) the expression

$$
E(N)=4|1-R(N)|<4\left|1-v_{1}(N)\right|=4(4(N+1)-2)^{-2},
$$

where the leading 4 is the scale factor from (14). 
Thus, by (15), expected errors for (14) are

$$
E(10)<2.3 \cdot 10^{-3}, E(300)<2.8 \cdot 10^{-6} \text { and } E(10000)=2.5 \cdot 10^{-9} \text {. }
$$

Verification: empirical errors of (14) are

$$
\operatorname{Pi}(10)-\pi=1.0 \cdot 10^{-3}, \operatorname{Pi}(300)-\pi=1.1 \cdot 10^{-6} \text { and } \operatorname{Pi}(10000)-\pi=1.0 \cdot 10^{-9} \text {. }
$$

\section{Acknowledgements}

The authors are indebted to Andrzej Majczak for writing this paper in the Word editing system.

\section{References}

[1] Wallis J., Arithmetica Infinitorum, Oxford 1656.

[2] Chabert J.-L. (ed.), A History of Algorithms: From the Pebble to the Microchip, Springer-Verlag, Berlin, Heidelberg, New York 1999.

[3] Arfken G.B., Weber H.J., Mathematical Methods for Physicists, Academic Press, San Diego, New York 1995.

[4] Berggren L., Borwein J., Borwein P. (eds.), Pi: A Source Book, Springer-Verlag, New York 2004.

[5] Kahlig P., Matkowski J., On the Dido functional equation, Ann. Math. Siles. 1999, 13, 167-180.

[6] Kahlig P., Matkowski J., Sharkovsky A.N., Dido's functional equation revisited, Rocznik Naukowo-Dydaktyczny Akademii Pedagogicznej w Krakowie, Prace Matematyczne 2000, 17, 143-150 .

[7] Courant R., Robbins H., Stewart I., What is Mathematics? Oxford University Press, Oxford 1996.

[8] van der Waerden B.L., Algebra I, Springer-Verlag, Berlin, Heidelberg, New York 1966. 